\title{
COMPUTATIONAL METHOD OF X-RAY HARMONIC ELIMINATION IN EXAFS TREATING
}

\author{
Ph.M. Ovsjannikov \\ Institute of Physics, Rostov State University \\ Stachky Ave. 194, R.ostov on Don, 344104, Russia
}

\begin{abstract}
The computational method of EXAFS amplitude correction by harmonics elimination and intensity leakage assessment has been worked out. In conventional absorption measurements, the major sources of errors are the transition of harmonics by the monochromator and inevitable leakage of radiation around and (or) through the sample. In this paper we present the method of the effective corrections being based on the measurements of the intensity of X-ray radiation passed through calibrated metal foils both without and together with investigated samples. As the advantage of the method one may emphasise the fact that it does not require any additional data for the investigated samples such as chemical composition, density, table absorption coefficients.
\end{abstract}

PACS numbers: 78.70.Dm, 87.64.Fb

\section{Introduction}

The effect of harmonic on EXAFS is one of the commonly met problem in the measurements. It is well known that the harmonics decrease the EXAFS amplitude as it has been discussed by some authors [1-3]. There are some methods of correction of these EXAFS signal distortions [1,2]. But these methods are based on the table data (for example [4]) and that is why they may be used for samples with a simple composition or with a known chemical composition and density. The presented method as composed of the existing ones requires only an additional exposition time and some additional calculations. It is useful for any composition of samples and does not require knowledge of any additional data for the investigated samples.

The main aim of the method is as usually the extraction of the fundamental absorption coefficient $\left(\mu_{j} d\right)$ from the measured one

$$
\mu d=\ln \left(I_{0} / I\right)
$$

where $\mu$ is a linear absorption coefficient, $d$ is a sample thickness, $I_{0}$ is an intensity of incident beam and $I$ is an intensity of that one passed through the sample. 
It is known that an incident $\mathrm{X}$-ray beam contains harmonics. The beam with wavelengths $\lambda_{i}=\lambda / i$ is reflected from a monocrystal monochromator at the Bragg angle $\theta=\arcsin \left(i \lambda / 2 d_{\mathrm{cr}}\right)$, where $d_{\mathrm{cr}}$ is a constant of the monocrystal, $i=1,2 \ldots k$. are the harmonic numbers, $\lambda_{1}$ is the minimum wa velength for $\theta$ and $d_{\mathrm{cr}}$. Not always the fundamental harmonic is the first one to be observed.

\section{Results and discussion}

\subsection{Preliminary step of the method}

This step is the common one for any investigated samples. At this step we obtain the normalised intensity $\alpha_{i}=I_{0}^{i} / I_{0}^{j}$ of the $i$-th harmonic in respect to the fundamental one $\left(j-t_{h}\right)$. We use metal foil with well known table mass absorption coefficient $\left(\tau_{m}^{i}\right)$, density $(\rho)$ and precisely $(0.01 \mathrm{~mm})$ measured thickness $(h)$. This precision of thickness measurements is sufficient to satisfy a statistical error of $I$ and $I_{0}(0.01 \%)$. We can write

$$
I=\sum_{i=1}^{k} I_{0}^{i} \exp \left(-\tau_{m}^{i} \rho h n\right)
$$

for the intensity of the bearn passed through absorber consisting of $n$ metal foils. The incident beam intensity $I_{0}$ may be also written as

$$
I_{0}=\sum_{i=1}^{k} I_{0}^{i}
$$

Substituting Eq. (2) and Eq. (3) to Eq. (1) and varying $n\left(d=h n_{g}\right)$ we get a system of linear equations for the intensity of harmonics $\alpha_{i}$

$$
\sum_{i \neq j}^{k-1} \alpha_{i}\left[1-\exp \left(-\tau_{m}^{i} \rho h n_{g}\right)\right]=\exp \left(\mu d-\tau_{m}^{j} \rho h n_{g}\right)-1,
$$

where $g \geq k-1$. The result of solving of the systern (4) is shown in Fig. 1 for Mo $K$-edge spectrum interval. In the present work we use an in-lab spectrometer [5] and an aluminum foil as absorber.

\subsection{General step}

The procedure of the preliminary step is repealed for the absorbed consisting of $n_{g}$ foils and together with the sample only at $5-10$ points of a spectrum before the absorption edge. In other words we substitute $I$ in Eq. (2) by

$$
I=\sum_{i=1}^{k} I_{0}^{i} \exp \left(-\tau_{m}^{i} \rho h . n\right) \exp \left(-\mu_{i} d\right) .
$$

This way we obtain the system of equations for the sample absorption coefficients at harmonic energies $\left(\mu_{i} d\right)$

$$
\sum_{i=1}^{k} \alpha_{i} \exp \left(-\tau_{m}^{i} \rho h n_{g}\right) \exp \left(-\mu_{i} d\right)=\sum_{i=1}^{k} \alpha_{i} \exp (-\mu d) .
$$



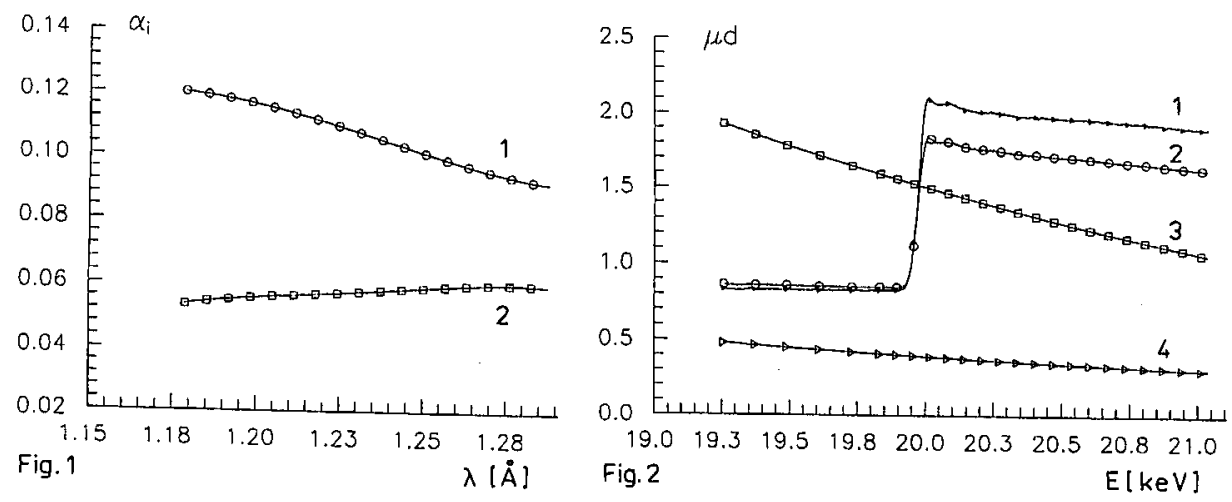

Fig. 1. The harmonic intensity spectra at the Mo $K$-edge EXAFS for the first harmonic $(i=1)$ - curve 1 - and for the third harmonic $(i=3)$ - curve 2. The second harmonic is the fundamental $(j=2)$. The scale of abscissa axis corresponds to the first harmonic.

Fig. 2 Energy dependencies of linear absorption coefficients for $\mathrm{H}_{2} \mathrm{MoO}_{4}$ at first (curve 3), third (curve 4) and fundamental (curve 1) harmonic spectrum interval. Curve 2 shows the experimental linear absorption coefficient before the separation harmonics. The abscissa scale corresponds to curves 1 and 2 .

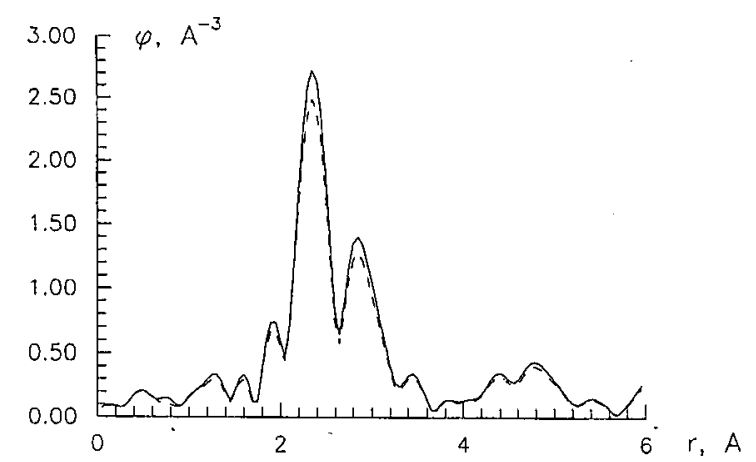

Fig. 3. Fourier transform of metal Mo before (dashed line) and after (solid line) only $6 \%$ harmonic elimination.

The terms with mass absorption coefficients refer to aluminum foils and the terms with linear absorption coefficients refer to the investigated sample. Solving the systern of Eqs. (6) and extrapolating evaluated coefficients to the whole spectrum interval using Victoreen's law we easily extract (Fig. 2) the fundamental absorption coefficient from the measured one by using the following equation:

$$
\mu_{j} d=\ln \left[\left(\sum_{i=1}^{k} \alpha_{i}\right) \exp (-\mu d)-\sum_{i=1}^{k-1} \alpha_{i} \exp \left(-\mu_{i} d\right)\right] .
$$

The result of the fundamental coefficient extraction for $\mathrm{H}_{2} \mathrm{MoO}_{4}$ is presented in Fig. 2. Figure 3 shows modifications obtained for Mo Fourier transform by har- 
monic elimination. The higher harmonics (above the fundamental one) produce more distortion of the EXAFS signal than the lower ones [1-3]. Only $6 \%$ of the third harmonic intensity (see Fig. 1) reduced the magnitude of the Fourier transform of Mo by $10 \%$. The result of this correction is shown in Fig. 3.

\section{Q.3. Corrections on the "sample holes"}

To assess the leakage of radiation through and (or) around the sample let us define the intensity of leakage $\mathrm{X}$-ray radiation as $y I_{0}$. We can rewrite Eq. (2) as

$$
I=y I_{0}+(1-y) \sum_{i=1}^{k} I_{0}^{i} \exp \left(-\tau_{m}^{i} \rho h n\right) .
$$

Then we repeat the above described general step. Additionally to the system (6) of equations we obtain the following equation for the intensity of leakage of X-ray radiation $y$ :

$$
\sum_{i=1}^{k-1} \alpha_{i}\left[1-\exp \left(-\tau_{m}^{i} \rho h n_{g}\right)\right]-\beta=\exp \left(\mu d-\tau_{m}^{j} \rho h n_{g}\right)-1,
$$

where $\beta=y \sum_{i=1}^{k} \alpha_{i}\left[1-\exp \left(-\tau_{m}^{i} \rho h n_{g}\right)\right] . y$ is equal to 0.04 for $\mathrm{H}_{2} \mathrm{MoO}_{4}$.

\section{Conclusions}

The method presented above allows an increase in the precision of EXAFS measurements by using only few additional measurements easy to perform. Commonly suggested methods with the use e.g. of total reflection Au mirrors or direct computational methods [1] require knowledge of the mass absorption coefficients for investigated substance and additional structural data (as exact chemical composition, density etc.). It is shown that the existence of high harmonics in the spectrum requires correction especially for samples with a high atomic number.

\section{References}

[1] J. Goulon, C. Goulon-Ginet, R. Cortes, J.M. Dubois, J. Phys. (France) 43, 539 (1983).

[2] E.A. Stern, K. Kim, Phys. Rev. B 23, 3781 (1981).

[3] L. Kunquan, W. Jun, Z. Yagin, C. Longain, F. Zhengzhi, Physica B 158, 273 (1989).

[4] W.H. McMaster, N. Kerr del Grande, J.H. Mallett, J.H. Hubbell, Compilation of X-Ray Cross Sections, Lawrence Rad. Lab., Report No. UCRL-50174, 1969.

[5] A.T. Shuvaev, B. Yu. Helmer, T.A. Lyubeznova, Pribori i Techn. Experim. 3, 234 (1988). 